\title{
Hereditary Factor X Deficiency
}

National Cancer Institute

\section{Source}

National Cancer Institute. Hereditary Factor X Deficiency. NCI Thesaurus. Code C98940.

A rare autosomal recessive inherited blood coagulation disorder characterized by deficiency of factor $X$, resulting in bleeding. 